Supporting Information for

\title{
Ultrahighly Photosensitive and Highly Stretchable Rippled Structure Photodetectors Based on Perovskite Nanocrystals and Graphene
}

Ying-Huan Chen ${ }^{\dagger}$, Monika Kataria ${ }^{\dagger \ddagger} \|$, Hung-I Lin ${ }^{\dagger}$, Christy Roshini Paul Inbaraj ${ }^{\dagger} \perp^{\#}, Y_{\text {- }}$ Ming Liao ${ }^{\dagger}$, Han-Wen Hu ${ }^{\dagger}$, Ting-Jia Chang ${ }^{\dagger}$, Cheng-Hsin Lu ${ }^{\nabla}$, Wei-Heng Shih ${ }^{\nabla}$, Wei-Hua Wang $^{\S}$, and Yang-Fang Chen *i。

$\dagger$ Department of Physics, National Taiwan University, Taipei 106, Taiwan

$\ddagger$ Department of Physics, National Central University, Chung-Li 320, Taiwan

$\S$ Molecular Science and Technology Program, Taiwan International Graduate Program, Institute of Atomic and Molecular Sciences, Academia Sinica, Taipei 115, Taiwan

" Institute of Atomic and Molecular Sciences, Academia Sinica, Taipei 115, Taiwan

$\perp$ Nanoscience and Nanotechnology Program, Taiwan International Graduate Program, Institute of Physics, Academia Sinica, Taipei 106, Taiwan

\# Department of Engineering and System Science, National Tsing Hua University, Hsinchu 300, Taiwan.

$\nabla$ Department of Materials Science and Engineering, Drexel University, Philadelphia, PA 19104, USA

${ }^{\circ}$ Advanced Research Centre for Green Materials Science and Technology, National Taiwan University, Taipei 10617, Taiwan

*Corresponding author: Yang-Fang Chen yfchen@phys.ntu.edu.tw

13 Pages, 10 Figures, 1 Table 


\section{SI. Fabrication method of perovskite nanocrystals and graphene rippled devices}

In order to fabricate the perovskite nanocrystals/graphene hybrid rippled structure photodetectors, the following steps were performed:

Step 1. A poly(dimethylsiloxane) (PDMS) film was stretched twice its length (100\% lateral strain) on a glass substrate.

Step 2. On the pre-strained PDMS, graphene with a layer of poly(methyl methacrylate) (PMMA) was transferred using the flipping technique.

Step 3. Silver electrodes were deposited on graphene/PMMA/PDMS using thermal vapor deposition technique.

Step 4. Perovskite Nanocrystals were spin coated on the graphene transferred on the prestrained PDMS substrate.

Step 5. Pre-strained PDMS was then released in order to get hybrid rippled structure photodetector.

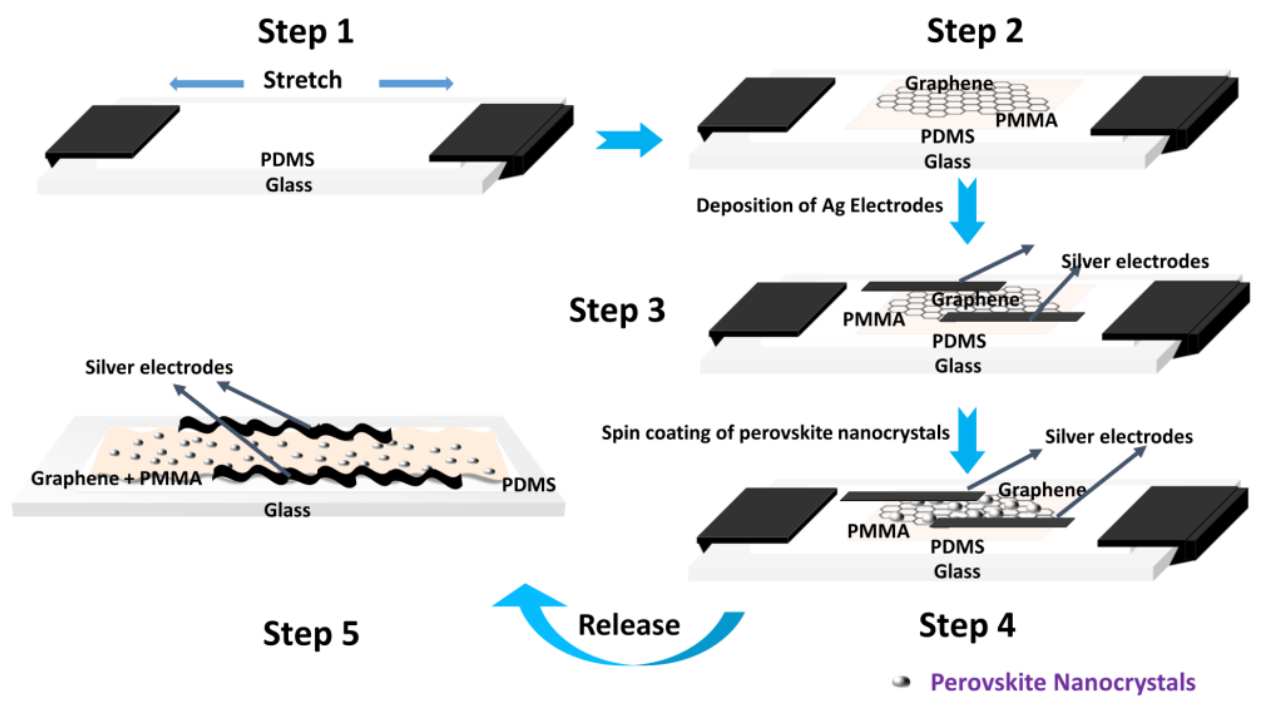

Figure S1. Schematic diagram for the fabrication of hybrid perovskite/graphene rippled structure photodetector. 


\section{SII. Definition of lateral strain}

Lateral strain is defined as the strain applied in the direction of plane parallel to the surface of PDMS film. When a PDMS film is stretched twice its original length along the direction of force applied in stretching it, it is defined as $100 \%$ strain. The original length of the PDMS film corresponds to $0 \%$ strain. Here, the device was fabricated such that the original length of the PDMS film taken was $2 \mathrm{~cm}$. It was then stretched upto $4 \mathrm{~cm}$ in length in order to apply $100 \%$ strain.

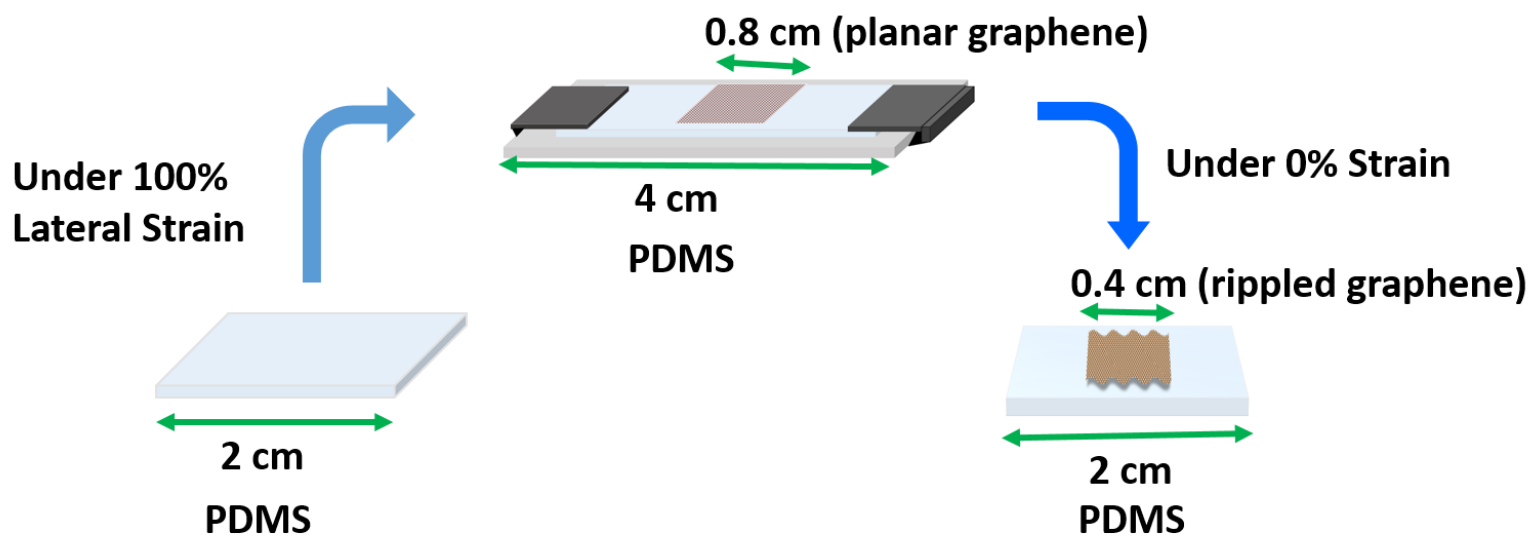

Figure S2. Schematic diagram for the definition of lateral strain. 


\section{SIII. Optimization process for optimal amount of perovskite nanocrystals coated over rippled graphene/PMMA structure for better photoresponse}

In order to get the best photoresponse from our hybrid rippled structure photodetector, an optimization process was carried out. This process included optimizing the layer of perovskite nanocrystals on the graphene. Different rotations per minute variations were incorporated in order to obtain different thickness of layer of perovskite nanocrystals over graphene. A hybrid planar structure photodetector with channel length $45 \mu \mathrm{m}$ (see Figure S3b) was fabricated to carry out the optimization process. The different rotations per minute variations undertaken include: (a) 1000 (5 seconds): 1500 (25 seconds), (b) 1000 (5 seconds): 2500 (25 seconds), (c) 1000 (5 seconds): 3500 ( 25 seconds), and (d) 1000 (5 seconds): 4500 (25 seconds). Figure S3a shows the photocurrent versus rotations per minute (RPM) variation of the perovskite nanocrystals of the hybrid planar structure photodetector at $\mathrm{V}_{\mathrm{DS}}=0.1 \mathrm{~V}$ and $4.8 \mathrm{~W} / \mathrm{m}^{2}$ of 325 nm laser illumination. It was observed that the highest photocurrent was observed in the case of 1000 ( 5 seconds): 2500 ( 25 seconds) rpm variation. The photocurrent is lesser for the rest of the variations. For the perovskite nanocrystals coated over graphene, photocurrent is maximum following the 1000 ( 5 seconds): 2500 (25 seconds) parameter, which is due to the fact that the penetration depth suits best for photogenerated charge carriers for this case. The perovskite nanocrystals coated using this parameter resulted in a layer thickness of $\sim 150 \mathrm{~nm}$. The characteristic diffusion length for the electrons and the holes is about $130 \mathrm{~nm}$ and $110 \mathrm{~nm}$. This helped in a faster transfer of photogenertaed charge carriers from the perovskite nanocrystals layer to graphene in comparison to the rest of the cases. The rest of the cases resulted in either a relatively thicker or a thinner layer of perovskite nanocrystals. A thicker layer provided for trapping center for photogenertaed charge carriers which lessens the photoresponse from the device. A thinner layer reduced the absorption of photons by the perovskite nanocrystals layer 
which in turn reduces the number of photogenertaed charge carriers. This resulted in a poorer photoresponse from the device as the thickness layer of perovskite nanocrystals reduces. So, overall an optimized layer thickness of perovskite nanocrystals was important and was experimentally found to be $\sim 150 \mathrm{~nm}$.

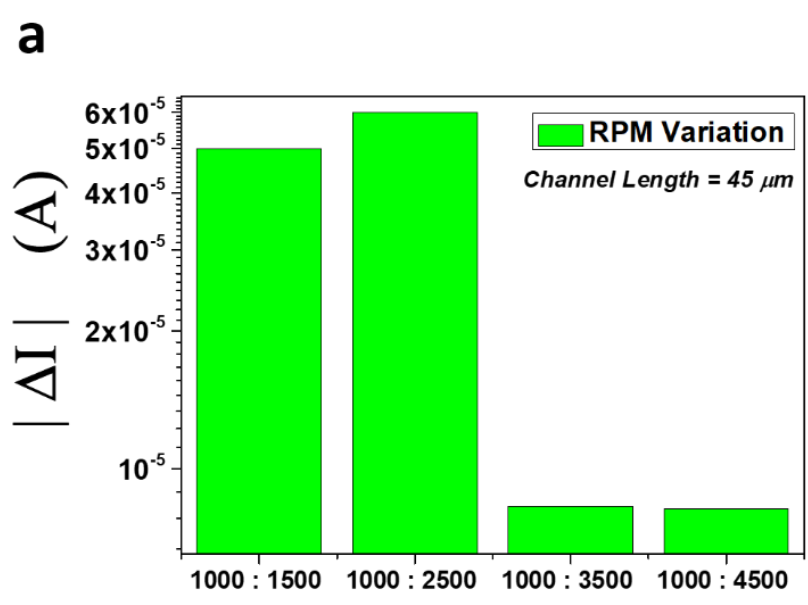

b

(RPM 1 : RPM 2) ; (5 seconds : 25 seconds)

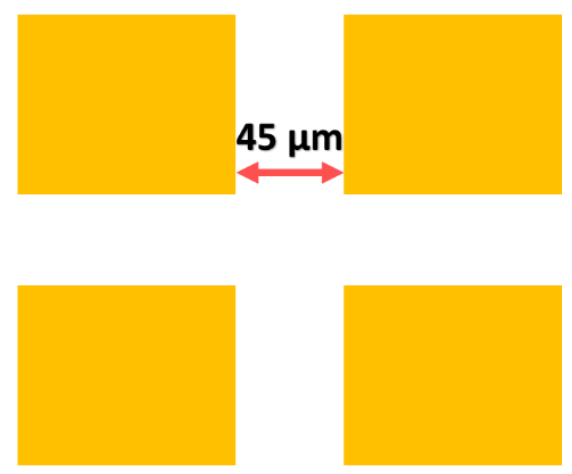

Figure S3. (a) Photocurrent versus rotations per minute (RPM) variation of the perovskite nanocrystals of the hybrid planar structure photodetector. (b) Schematic diagram to show the channel length of the device used for optimization process. 


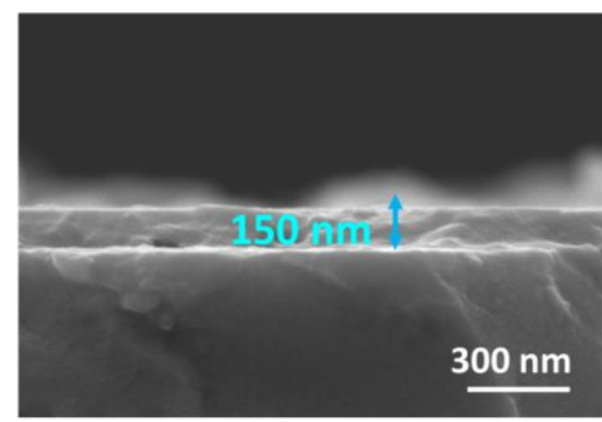

Figure S4. Cross-sectional Scanning Electron Microscopy (SEM) image of the film of perovskite nanocrystals on silicon substrate.

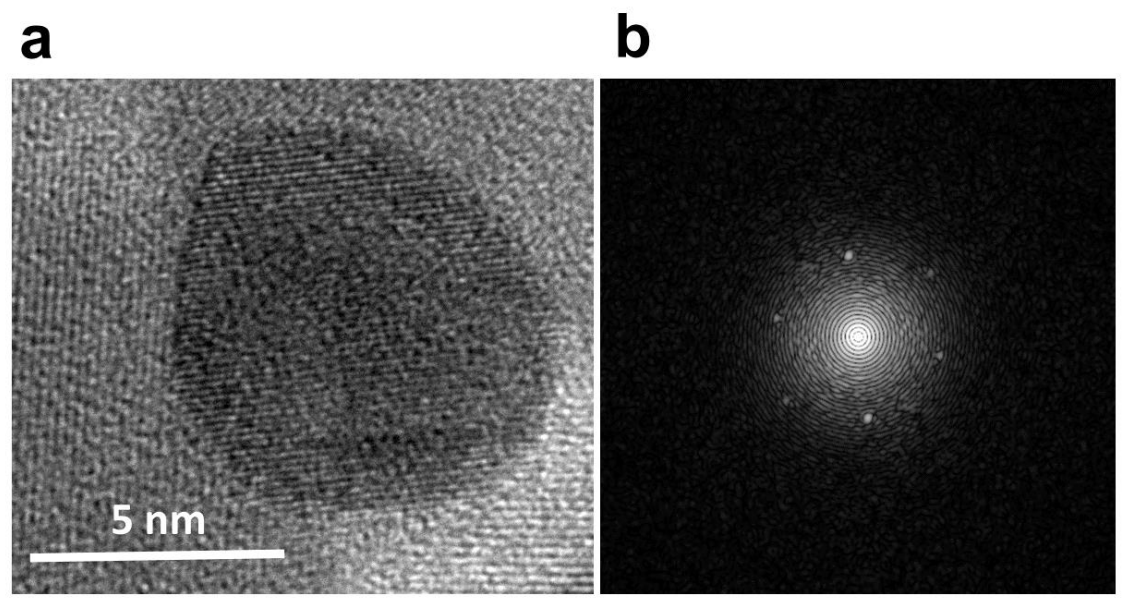

Figure S5. (a) Transmission Electron Microscopy (TEM) image of a single perovskite nanocrystal. (b) Fast Fourier transform (FFT) pattern of perovskite nanocrystals. 
(a)

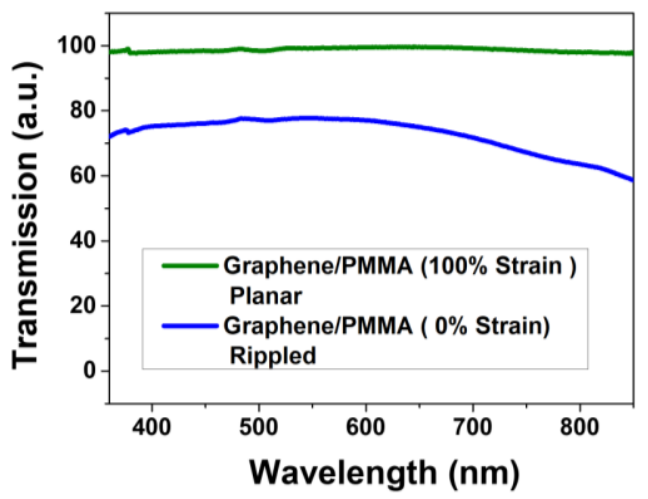

(b)

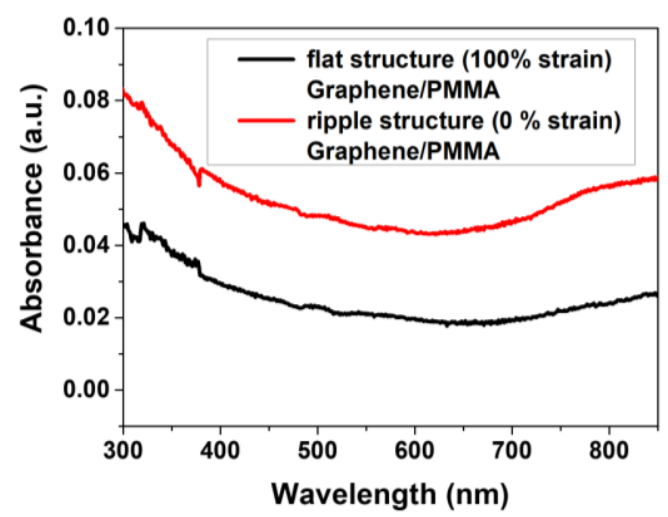

Figure S6. (a) Transmission spectra of graphene/PMMA under $100 \%$ lateral strain and $0 \%$ lateral strain. (b) Absorbance spectra of flat structure (graphene/PMMA) and rippled structure (graphene/PMMA).

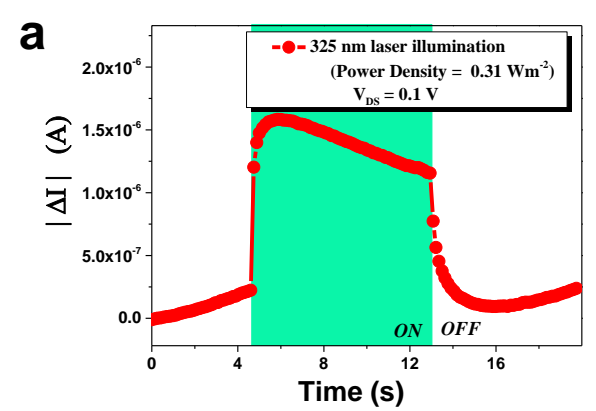

b

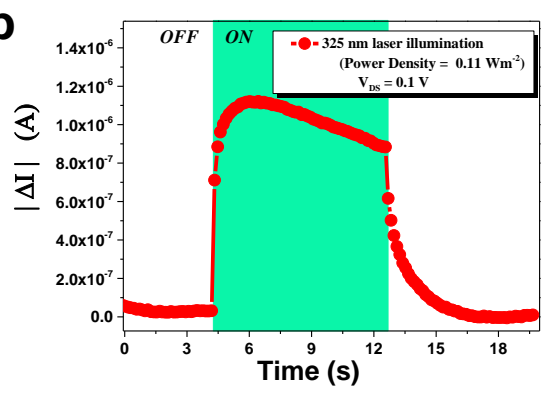

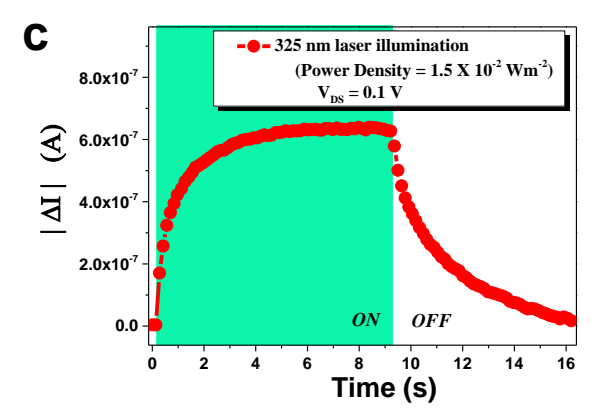

d

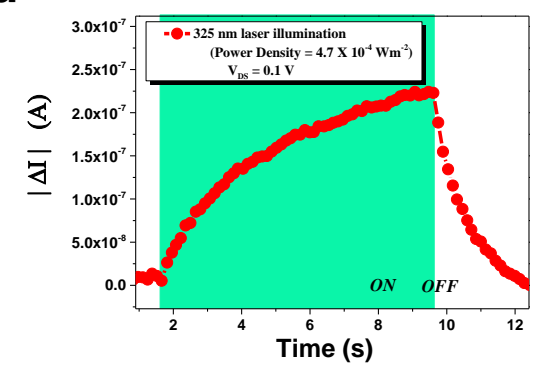

e

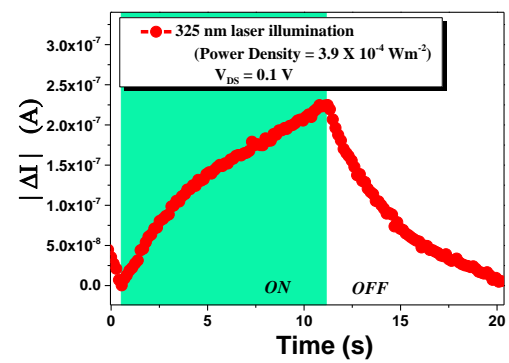

Figure S7. The photocurrent of the device under various optical power densities of laser irradiance (a) $0.31 \mathrm{Wm}^{-2}$, (b) $0.11 \mathrm{Wm}^{-2}$, (c) $1.5 \times 10^{-2} \mathrm{Wm}^{-2}$, (d) $4.7 \times 10^{-4} \mathrm{Wm}^{-2}$, and (e) 3.9 $\times 10^{-4} \mathrm{Wm}^{-2}$ of $325 \mathrm{~nm}$ laser illumination. 


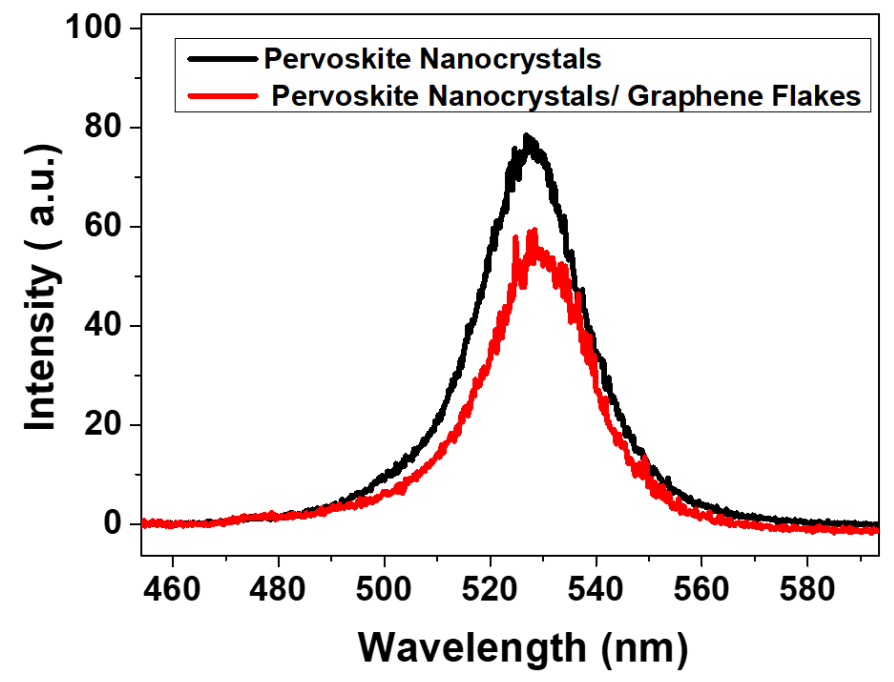

Figure S8. Photoluminescence Spectra of perovskite nanocrystals and perovskite nanocrystals/graphene under $374 \mathrm{~nm}$ laser excitation.

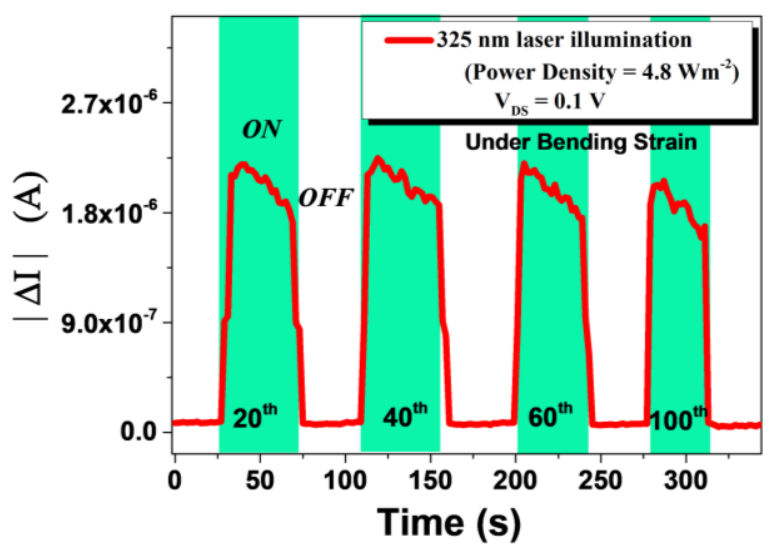

Figure S9. The photocurrent of the device with $0 \%$ strain subjected to different cyclic stretching of $2 \%$ bending strain under laser irradiance with $325 \mathrm{~nm}$ wavelength. 

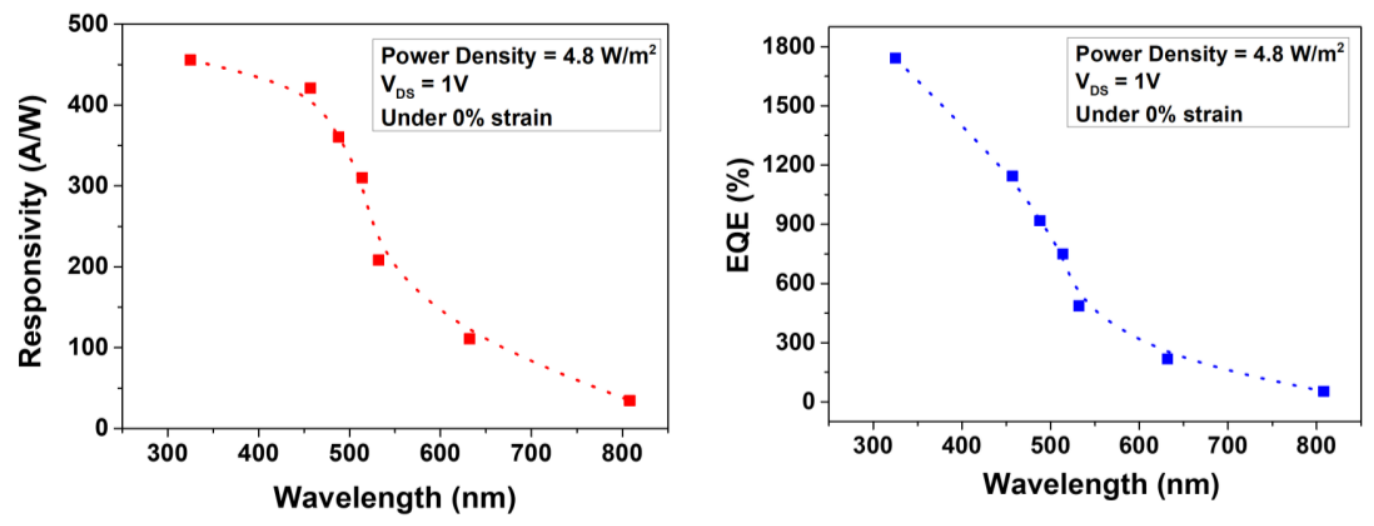

Figure S10. (a) Dependence of responsivity on various wavelength of laser irradiance. (b) Dependence of EQE on various wavelength of laser irradiance. 
Table SI. Table for comparing responsivities of different similar kind of devices

\begin{tabular}{|c|c|c|}
\hline S. No. & $\begin{array}{l}\text { Device Composition } \\
\text { (Rippled Structure) }\end{array}$ & $\begin{array}{c}\text { Device } \\
\text { Performance }\end{array}$ \\
\hline 1 & Perovskite nanocrystals/graphene/PMMA/PDMS ( Channel length $=100 \mu \mathrm{m})$ & $\begin{array}{l}\sim 6{\mathrm{X} 10^{5} \mathrm{~A} \mathrm{~W}^{-1}}^{\text {This Work }}\end{array}$ \\
\hline 2 & Graphene/ Very High Bond (VHB) substrate ${ }^{\mathrm{S} 1}$ & $0.011 \mathrm{~mA} \mathrm{~W}^{-1}$ \\
\hline 3 & Graphene/ Gold Nanoparticles/ VHB substrate ${ }^{\mathrm{S} 2}$ & $0.044 \mathrm{~mA} \mathrm{~W}^{-1}$ \\
\hline 4 & Upconversion nanoparticles/graphene/PMMA/PDMS ( Channel length $=100 \mu \mathrm{m}){ }^{\mathrm{S} 3}$ & $26 \mathrm{~A} \mathrm{~W}^{-1}$ \\
\hline 5 & Metal-Organic framework/graphene/PMMA/PDMS ( Channel length $=100 \mu \mathrm{m}$ ) ${ }^{\mathrm{S} 4}$ & $8{\mathrm{X} 10^{3} \mathrm{~A} \mathrm{~W}^{-1}}^{-1}$ \\
\hline 6 & Graphene quantum dots/ graphene/PDMS S5 & $7 \times 10^{2} \mathrm{~A} \mathrm{~W}^{-1}$ \\
\hline 7 & $\mathrm{CH}_{3} \mathrm{NH}_{3} \mathrm{PbI}_{3}$ microwires/ graphene/ VHB Film ${ }^{\mathrm{S} 6}$ & $2.2 \mathrm{~mA} \mathrm{~W}^{-1}$ \\
\hline
\end{tabular}




\section{SIV. Experimental methods}

\section{Single-Layer Graphene Synthesis}

The detailed synthesis process can be found in our previously published reports. ${ }^{\mathrm{S} 3}$ In brief, monolayer graphene was synthesized on $99.99 \%$ pure copper substrate by using a standard chemical vapor deposition (CVD) technique. The 99.98\% pure copper foil from Aldrich was used, and it was first polished by electrolysis of $85 \%$ phosphoric acid (H3PO4) at $1.5 \mathrm{~V}$ for 15 min. When polishing the copper foil, another copper foil was used as a counter electrode to avoid the doping of the host atom into the copper. After the copper foil was polished, we could grow graphene on it. The polished copper foil was placed into the CVD furnace for $60 \mathrm{~min}$ at $1000{ }^{\circ} \mathrm{C}$ with $60 \mathrm{SCCM}$ of $\mathrm{H}_{2}$ flow. Next, $3.4 \mathrm{SCCM}$ of $\mathrm{CH}_{4}$ was made to flow through the CVD furnace for $30 \mathrm{~min}$, with the same above-mentioned parameters. The chemical reaction between $\mathrm{H}_{2}$ and $\mathrm{CH}_{4}$ at $1000{ }^{\circ} \mathrm{C}$ led to the deposition of a single layer of graphene on both sides of the polished copper. To transfer the graphene onto the PDMS substrate, PMMA over graphene/ $\mathrm{Cu}$ was spin coated. Then, graphene/ $\mathrm{Cu}$ was made to float in $\mathrm{FeCl}_{3}$ solution to etch the copper. Next, PMMA/graphene was transferred onto distilled water to wash out the solution $\mathrm{FeCl}_{3}$ from it. Lastly PMMA/graphene was flipped and transferred on PDMS substrate to get the structure of graphene/ PMMA/PDMS.

\section{Electrode Deposition}

The shadow mask used is G400 copper mesh (channel length $=45 \mu \mathrm{m})$ (Figure S3) and channel length $100 \mu \mathrm{m}$ (Figure 3a(i)) from Electron Microscopy Science. Ag electrodes, patterned by the mask, were deposited on the graphene/PMMA/ PDMS structure by a thermal evaporator. For the detailed process, it can be found in our published report. ${ }^{\mathrm{S} 3}$ 


\section{PDMS Synthesis}

The PDMS solution from Dow Corning Sylgard 184 was mixed with a cross-linking solution in a 10:1 ratio. The solution was then poured into a Petri dish and extended uniformly. It was then held in a furnace at $70{ }^{\circ} \mathrm{C}$ for $120 \mathrm{~min}$. Thus, a flexible thin film of PDMS was obtained. The synthesis process is similar to our published report. ${ }^{\mathrm{S} 3}$

\section{Perovskite Nanocrystals Synthesis}

To begin with, $96 \mu \mathrm{L}$ of oleic acid was added into $2 \mathrm{~mL}$ of octadecene. The solution was then heated and stirred at $80^{\circ} \mathrm{C}$. Then $0.06 \mathrm{mmol}$ of octylammonium bromide (Shanghai MaterWin New Materials) and $0.1 \mathrm{mmol}$ of lead (II) bromide were then next added. The key note here is to make sure the previous precursor is fully dissolved before adding the subsequent precursor. After 5 min reaction, a yellow dispersion was produced. The obtained dispersion was purified by adding acetone to precipitate the nanoparticles, followed by centrifugation at $3000 \mathrm{rpm}$ for 10 min, discarded the supernatant, and redispersed the precipitates in toluene for storage and further usage. The as synthesized perovskite nanocrystals have a strong photoluminescence as shown in Figure S8. For more detailed description, please refer to our published work. ${ }^{\mathrm{S} 7}$ 


\section{References}

(S1) Kang, P.; Wang, M. C.; Knapp, P. M.; Nam, S. Crumpled Graphene Photodetector with Enhanced, Strain-Tunable, and Wavelength-Selective Photoresponsivity. Adv. Mater. 2016, 28, 4639-4645.

(S2) Kim, M.; Kang, P.; Leem, J.; Nam, S. A Stretchable Crumpled Graphene Photodetector with Plasmonically Enhanced Photoresponsivity. Nanoscale 2017, 9 , 4058-4065.

(S3) Kataria, M.; Yadav, K.; Haider, G.; Liao, Y. M.; Liou, Y.-R.; Cai, S.-Y.; Lin, H.-i.; Chen, Y. H.; Paul Inbaraj, C. R.; Bera, K. P. Transparent, Wearable, Broadband, and Highly Sensitive Upconversion Nanoparticles and Graphene-Based Hybrid Photodetectors. ACS Photonics 2018, 5, 2336-2347.

(S4) Bera, K. P.; Haider, G.; Usman, M.; Roy, P. K.; Lin, H. I.; Liao, Y. M.; Inbaraj, C. R. P.; Liou, Y. R.; Kataria, M.; Lu, K. L. Trapped Photons Induced Ultrahigh External Quantum Efficiency and Photoresponsivity in Hybrid Graphene/Metal-Organic Framework Broadband Wearable Photodetectors. Adv. Funct. Mater. 2018, 28, 1804802.

(S5) Chiang, C.-W.; Haider, G.; Tan, W.-C.; Liou, Y.-R.; Lai, Y.-C.; Ravindranath, R.; Chang, H.-T.; Chen, Y.-F. Highly Stretchable and Sensitive Photodetectors based on Hybrid Graphene and Graphene Quantum Dots. ACS Appl. Mater. Interfaces 2016, 8, 466-471.

(S6) Ding, J.; Fang, H.; Lian, Z.; Lv, Q.; Sun, J.-L.; Yan, Q. High-Performance Stretchable Photodetector based on $\mathrm{CH}_{3} \mathrm{NH}_{3} \mathrm{PbI}_{3}$ Microwires and Graphene. Nanoscale 2018, 10, 10538-10544.

(S7) Hu, H. W.; Haider, G.; Liao, Y. M.; Roy, P. K.; Ravindranath, R.; Chang, H. T.; Lu, C. H.; Tseng, C. Y.; Lin, T. Y.; Shih, W. H. Wrinkled 2D Materials: A Versatile Platform for Low-Threshold Stretchable Random Lasers. Adv. Mater. 2017, 29, 1703549. 